\title{
Primer reporte de Haemaphysalis juxtakochi Cooley, 1946 (Acari: Ixodidae) parasitando equinos en Uruguay
}

\author{
Félix $\mathrm{ML}^{1}$, Venzal $\mathrm{JM}^{1^{*}}$ \\ ${ }^{1}$ Laboratorio de Vectores y Enfermedades Transmitidas, Facultad de Veterinaria, CENUR Litoral \\ Norte - Salto, Universidad de la República. \\ * Correspondencia: José M. Venzal. Facultad de Veterinaria, CENUR Litoral Norte, \\ Rivera 1350, 50000 Salto, Uruguay. \\ E-mail: jvenzal@unorte.edu.uy
}

Recibido: 30 de Marzo 2019. Aceptado: 15 Abril 2019. Disponible en línea: 16 Abril 2019

Editor: S. Nava

RESUMEN. Se presenta el primer reporte de Haemaphysalis juxtakochi Cooley, 1946 parasitando equinos en Uruguay. Adultos de esta especie de garrapata fueron hallados en un equino junto a Rhipicephalus microplus (Canestrini, 1888) en una localidad del departamento de Tacuarembó, con presencia de monte nativo denso y abundante fauna silvestre, como el ciervo guazubirá (Mazama gouazoubira), hospedador preferencial de adultos de $H$. juxtakochi. Con este hallazgo son cuatro las especies de garrapatas parásitas de equinos reportadas para Uruguay: $R$. microplus, Amblyomma tigrinum Koch, 1844, Amblyomma triste Koch, 1844 y H. juxtakochi. La importancia médico-veterinario de $H$. juxtakochi en equinos es desconocida.

SUMMARY. First report of Haemaphysalis juxtakochi Cooley, 1946 (Acari: Ixodidae) parasitizing horses in Uruguay. The first report of Haemaphysalis juxtakochi Cooley, 1946 parasitizing horses in Uruguay is presented. Adults of this tick species were found in an equine together with Rhipicephalus microplus (Canestrini, 1888), in a locality of the department of Tacuarembó with presence of dense native forest and wildlife abundant, as well as the gray-brocket deer (Mazama gouazoubira) preferential host of $H$. juxtakochi adults. With this finding there are four species of ticks that can be found parasitizing equines in Uruguay: $R$. microplus, Amblyomma tigrinum Koch, 1844, Amblyomma triste Koch, 1844 and $H$. juxtakochi. The medical-veterinary importance of $H$. juxtakochi in equines is unknown.

Palabras clave: Ixodidae, Haemaphysalis juxtakochi, Uruguay, equinos

Key words: Ixodidae, Haemaphysalis juxtakochi, Uruguay, horses

Las garrapatas son ectoparásitos hematófagos de una amplia variedad de vertebrados. Pueden causar en sus hospedadores: dermatosis, anemia, toxemia, parálisis, disminución del peso corporal y facilitar la aparición de miasis e infecciones bacterianas secundarias. Aunque el problema más importante asociado a las garrapatas es la transmisión de agentes patógenos como: virus, bacterias, protozoos y nematodos, a animales y humanos, ya que luego de los mosquitos son los artrópodos vectores más importantes de enfermedades infecciosas (Sonenshine y Roe, 2014). Actualmente unas 950 especies de garrapatas son consideradas válidas, siendo Haemaphysalis Koch 1844, con 167 especies descriptas, el segundo género de garrapatas con mayor número de especies ( $23 \%$ del total mundial) (Du et al., 2018; Guglielmone y Nava, 2014; Guglielmone y Robbins, 2018; Guglielmone et al., 2014; Mans et al, 2019). Una reciente revisión de las garrapatas del Cono Sur de América, lista unas 62 especies, de las que solo dos especies de Haemaphysalis son consideradas como establecidas: Haemaphysalis leporispalustris (Packard, 1869) y Haemaphysalis juxtakochi Cooley, 1946 (Nava et al, 2017). En Uruguay, se han reportado unas 20 especies de garrapatas, siendo hasta el momento: Rhipicephalus microplus (Canestrini, 1888), Rhipicephalus sanguineus (Latreille, 1806), Amblyomma aureolatum (Pallas, 1772) y Amblyomma triste Koch, 1844, las especies de mayor importancia en salud pública y veterinaria (Venzal et al, 2003, 2004; Martins et al, 2014; Nava et al, 2017, 2018). Los reportes de garrapatas parasitando a equinos en Uruguay son escasos, y las únicas especies documentadas son $R$. microplus, Amblyomma tigrinum Koch, 1844 y A. triste (Venzal et al, 2003; Martins et al, 2014). La piroplasmosis equina es causada por Babesia caballi (Nuttall and Strickland, 1910) y Theileria equi 
(Laveran, 1901) y transmitida por garra-patas, posee un importante impacto económico en la industria equina. Estas pérdidas económicas incluyen costos por tratamientos veterinario, disminución del rendimiento y muerte de animales. Además es una limitante para la exportación de equinos así como la participación en eventos deportivos (Díaz-Sánchez et al, 2018). En Uruguay la piroplasmosis equina es poco conocida. La primera comunicación de un caso clínico (re-portado como Nuttalliosis) corresponde a Cassamagnaghi y Cassamagnaghi (1948), quienes identifican como agente causal a Nuttallia equi (= Theileria equi). Más recientemente se ha confirmado la presencia de $T$. equi mediante diagnóstico serológico y molecular (PCR) (Solari, 2008). Si bien se sospecha que la transmisión de la piroplasmosis a los equinos en Uruguay está relacionada con $R$. microplus, no existen estudios al respecto. El objetivo del presente trabajo es reportar por primera vez la presencia de $H$. juxtakochi parasitando equinos para Uruguay.

Durante muestreos mensuales sobre ecología de garrapatas llevados a cabo en el noreste de Uruguay, el 11 de diciembre de 2017 en un establecimiento rural, próximo a Gruta de los Cuervos ( $\left.31^{\circ} 37^{\prime} 08^{\prime \prime} \mathrm{S}, 56^{\circ} 02^{\prime} 47^{\prime \prime} \mathrm{O}\right)$, departamento de Tacuarembó, se colectaron garrapatas parasitando a un equino. El encargado del establecimiento dio conocimiento a los autores de que los animales habitualmente estaban parasitados por garrapatas, sobre todo en zonas de pelos gruesos, como crines. Las garrapatas fueron conservadas en frascos tapa rosca con alcohol $95^{\circ}$ hasta su procesamiento en el laboratorio donde se identificaron morfológicamente siguiendo a Nava et al (2017) utilizando una lupa estereoscópica binocular Nikon SMZ 1000.

Como resultado, se colectaron un total de 22 garrapatas, 16 hembras de $H$. juxtakochi, y 5 hembras y 1 macho de R. microplus, las cuales fueron depositadas en la colección del Departamento de Parasitología Veterinaria (Facultad de Veterinaria, CENUR Litoral Norte. Salto, Uruguay) con números de acceso: DPVURU 902-903. En el caso de $R$ microplus, en nuestra región se debe diferenciar esta especie de $R$. sanguineus s.l., especie que, aunque tiene todos sus estadios asociados principalmente a caninos domésticos, en ocasiones puede ser hallada parasitando equinos, aunque no hay registros publicados de parasitismo por esta especie en equinos para Uruguay (Nava et al, 2017; Venzal et al, 2003). Tanto en machos como en hembras los principales caracteres morfológicos diagnósticos y diferenciales con $R$. sanguineus s.l. son: ausencia de festones, formula dental $4 / 4$, surco anal apenas perceptible y coxa I con espinas cortas y romas. Para Haemaphysalis, se debe diferenciar entre $H$. juxtakochi y $\mathrm{H}$. leporispalustris. Morfológicamente, los adultos de $H$. juxtakochi se diferencian de los de $H$. leporispalustris por tener el hipostoma con fórmula dental 4/4, ausencia de proceso ventral en la base del capítulo, y presencia de una larga espina ventral retrógrada a nivel del III artejo de los palpos (Fig. 1). Además, $H$. leporispalustris tiene como hospedadores preferenciales a lepóridos del género Sylvilagus (Nava et al, 2017).

El parasitismo por $R$. microplus en equinos, especie cuyo ciclo de vida es de un solo hospedador (monofásico), es un hecho frecuente en nuestro país, pero hasta el momento no se había reportado el parasitismo por $H$. juxtakochi. Especie cuyos adultos parasitan principalmente ciervos (Cervidae), y eventualmente otros mamíferos, e inmaduros (larva y ninfa) a micro mamíferos terrestres y aves (Nava et al, 2017). En Uruguay los adultos han sido reportados en bovinos y en el ciervo guazubirá (Mazama gouazoubira) (Venzal et al, 2003). El ambiente de donde procede el equino parasitado se caracteriza por poseer denso monte nativo con presencia de fauna silvestre, como por ejemplo $M$. gouazoubira, uno de los hospedadores preferenciales de $H$. juxtakochi. Con este hallazgo son cuatro las especies de garrapatas parásitas de equinos reportadas para Uruguay: $R$. microplus, A. tigrinum, A. triste y $H$. juxtakochi. Si bien la importancia médico-veterinario de $H$. juxtakochi en equinos es desconocida, para otra especie de este género, Haemaphysalis longicornis Neumann 1901, se demostró experimentalmente que puede ser vector competente en la transmisión de B. equi (Ikadai et al., 2007).

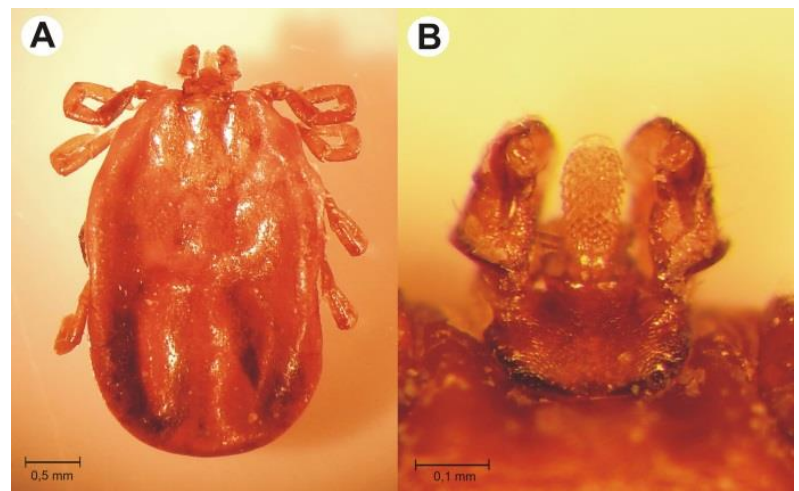

Figura 1. Haemaphysalis juxtakochi hembra. A) vista dorsal. B) capitulum ventral.

\section{Agradecimientos}

Al encargado y al dueño del establecimiento por colaborar con los estudios sobre ecología de garrapatas en el noreste de Uruguay.

\section{Bibliografía}

Cassamagnaghi A, Cassamagnaghi A (h). 1948. El primer caso de Nuttalliosis equina comprobado en Uruguay. An. Fac. Vet. Uruguay 2: 181-194.

Díaz-Sánchez AA, Fonseca-Rodríguez O, Castillo-Domínguez SL del, Alfonso-Dorta Y, Lobo-Rivero E, Corona-González B, VegaCañizares E. 2018. Alteraciones hematológicas encontradas en caballos (Equus caballus) infectados con Babesia caballi y Theileria equi. Rev. Salud Animal 40: 1-10. 
Du CH, Sun Y, Xu RM, Shao, ZT. 2018. Description of Haemaphysalis (Alloceraea) kolonini sp. nov., a new species in subgenus Alloceraea Schulze (Ixodidae: Haemaphysalis) in China. Acta Parasitol. 63: 678-691.

Guglielmone AA, Nava S. 2014. Names for Ixodidae (Acari: Ixodoidea): valid, synonyms, incertae sedis, nomina dubia, nomina nuda, lapsus, incorrect and suppressed names-with notes on confusions and misidentifications. Zootaxa 3767: 1-256.

Guglielmone AA, Robbins RG. 2018. Hard Ticks (acari: Ixodida: Ixodidae) Parasitizing Humans: A Global Overview. Springer Intl. publishing. $314 \mathrm{pp}$.

Guglielmone AA, Robbins RG, Apanaskevich DA, Petney TN, Estrada-Peña A, Horak IG. 2014. The hard ticks of the world (Acari: Ixodida: Ixodidae). Springer, London. 738 pp.

Ikadai H, Sasaki M, Ishida H, Matsuu A, Igarashi I, Fujisaki K, Oyamada T. 2007. Molecular evidence of Babesia equi transmission in Haemaphysalis longicornis. Am. J. Trop. Med. Hyg. 76: 694-697.

Mans BJ, Featherston J, Kvas M, Pillay KA, de Klerk DG, Pienaar R, de Castro MH, Schwan TG, Lopez JE, Teel P, Perez de Leon AA, Sonenshine DE, Egekwu NI, Bakkes DK, Heyne H, Kanduma, EG, Nyangiwe N, Bouattour A, Latif AA. 2019. Argasid and ixodid systematics: Implications for soft tick evolution and systematics, with a new argasid species list. Ticks Tick Borne Dis. 10: 219-240.

Martins TF, Lado P, Labruna MB, Venzal JM. 2014. El género Amblyomma (Acari: Ixodidae) en Uruguay: especies, distribución, hospedadores, importancia sanitaria y claves para la determinación de adultos y ninfas. Veterinaria (Montevideo) 50: 26-41.

Nava S, Venzal JM, González-Acuña D, Martins TF, Guglielmone AA. 2017. Ticks of the Southern Cone of America: Diagnosis, Distribution and Hosts with Taxonomy, Ecology and Sanitary Importance. Elsevier, Academic Press, London. 372 pp.

Nava S, Beati L, Venzal JM, Labruna MB, Szabo, MPJ, Petney TN, Saracho Bottero MN, Tarragona EL, Dantas-Torres F, Santos Silva MM, Mangold AJ, Guglielmone AA, Estrada-Peña, A. 2018. Rhipicephalus sanguineus (Latreille, 1806): Neotype designation, morphological re-description of all parasitic stages and molecular characterization. Ticks Tick Borne Dis. 9: 1573-1585.

Solari MA. 2008. Babesiosis equina: seroprevalencia, diagnóstico y control. Asociación Argentina de Veterinarios de Laboratorios de Diagnóstico - XVII Reunión Científica Técnica. Pp. 46-48.

Sonenshine DE, Roe RM. 2014. Overview. Ticks, people and animals. En: Sonenshine DE, Roe RM, editors. Tick biology. 2nd ed. Vol. 1. Oxford University Press, Oxford. Pp. 3-16.

Venzal JM, Castro O, Cabrera PA, De Souza CG, Guglielmone AA. 2003. Las garrapatas de Uruguay: especies, hospedadores, distribución e importancia sanitaria. Veterinaria (Montevideo). 38: $17-28$

Venzal JM, Portillo A, Estrada-Peña A, Castro O, Cabrera PA, Oteo JA. 2004. Rickettsia parkeri in Amblyomma triste from Uruguay. Emerg. Infect. Dis. 10: 1493-1495. 